\title{
A Chebyshev Minimax Technique Oriented to Aerospace Trajectory Optimization Problems
}

\author{
WiLliam F. Powers* \\ The University of Michigan, Ann Arbor, Mich.
}

\begin{abstract}
This paper describes a method based upon the classical calculus of variations for solving directly Chebyshev minimax problems which arise in trajectory optimization. The close relationship between minimax problems and problems with state variable inequality constraints is used to gain insight into the minimax problem, and to define an order for minimax functions. The method is applicable to 1$)$ all problems in which the first time derivative of the minimax function does not contain control variables explicitly, and 2) all problems with a "flat" maximum (including problems in which the first time derivative of the minimax function contains control variables). The theory is applied to both a simple example and a formulation of the optimal re-entry heating problem whose performance index consists of a minimax term for maximum heating rate, a terminal function for maximum crossrange, and a path integral for minimum total heating. Several shooting and gradient-type numerical algorithms are suggested by the approach.
\end{abstract}

\section{Introduction}

A BOLZA problem with interior point conditions is defined which allows the direct treatment of minimax problems. Other approaches to the minimax problem exist which either are concerned with problems that are mainly influenced by the control constraints ${ }^{1}$ or are approximate techniques which are either overly restrictive ${ }^{2}$ or involve the definition of auxiliary performance indices. ${ }^{3,4}$ The approach of this paper is oriented to problems that arise in trajectory optimization, in which case control variable inequality constraints are not the main influence in the problem (e.g., problems with few, if any, bounded control subarcs). Although the approach is different than the analyses by J. Warga in Refs. 5 and 6, the analogy with state-constrained problems noted by Warga is strongly evident here, also.

In Ref. 1 an extensive bibliography of the literature on minimax problems is presented. The literature refers to two different types of problems as minimax problems. a) Problems in which two controls are involved, with one of the controls attempting to minimize the same performance index that the other control is attempting to maximize (e.g., pursuit-evasion problems with the pursuer attempting to minimize distance while the evader is attempting to maximize distance. ${ }^{7}$ b) Problems in which the minimum of the maximum value of a function defined on $\left[t_{0}, t_{f}\right]$ is desired. This case is shown in Figs. 1 and 2 , where $F[x(\bar{t})]$ is to be minimized. In Fig. 1, a unique maximum is the case, while Fig. 2 shows the case in which $F[x(\bar{t})]$ is nonunique (or possesses a flat maximum).

Figures 1 and 2 indicate that a close relationship may exist between problems with state variable inequality constraints (SVIC) and minimax problems of type $b$ above. This is indeed the case, and the remainder of the paper will be concerned with the type $b$ minimax problem, which C. D. Johnson ${ }^{1}$ calls the "Chebyshev Minimax" (or C-minimax) problem.

In Ref. 8, Denham developed a method for treating problems with intermediate point constraints (e.g., staging) and state variable inequality constraints by forming the first variation of an augmented functional which includes these effects. A more complete discussion of this approach is given in Chap. 3 of Ref. 7 .

Received October 15, 1971; revision received May 3, 1972. This research area was suggested by personnel in the NASA Manned Spacecraft Center, Mission Planning and Analysis Division, and was supported in part by the National Science Foundation under Grant GK-30115.

Index categories: Navigation, Control, and Guidance Theory; Spacecraft Navigation, Guidance, and Flight-Path Control Systems.

* Associate Professor, Department of Aerospace Engineering. Member AIAA.

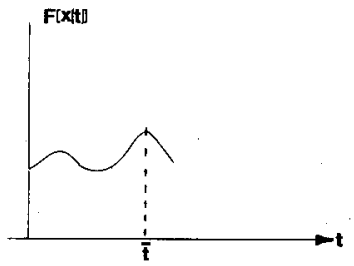

Fig. 1 Minimax with a unique maximum.

The same procedure can be applied to the minimax problem. As will be shown, the case of Fig. 1 has necessary conditions which are similar to those of an SVIC problem in which the constraint is active at a single point only. The case of Fig. 2, moreover, has necessary conditions which are similar to those of an SVIC problem in which the constraint is active over a nonzero time interval.

To gain insight into the approach, let us first consider the somewhat restricted case in which the optimal trajectorycontrol pair $\left\{x^{*}(t), u^{*}(t)\right\}$ is of continuity class $C^{2}\left[t_{0}, t_{f}\right]$. Assuming a normal problem, consider a one-parameter family of nearby trajectories and controls defined by $x(t, \varepsilon), u(t, \varepsilon)$, with $x^{*}(t)=x(t, 0), u^{*}(t)=u(t, 0)$. Let $\bar{t}^{*} \varepsilon\left(t_{0}, t_{f}\right)$ be a point on $x^{*}(t)$ at which the maximum of $F[x(t)]$ occurs. Then, it is necessary that

$$
\left.\dot{F}^{*}\right|_{\bar{t}^{*}} \equiv F_{x}^{T}\left[x^{*}\left(\bar{t}^{*}\right)\right] f\left[\bar{t}^{*}, x^{*}\left(\bar{t}^{*}\right), u^{*}\left(\bar{t}^{*}\right)\right]=0
$$

Note that, by the definition of the point $\bar{t}(\varepsilon)$, on every admissible trajectory in a sufficiently small neighborhood of $x^{*}(t)$

$$
\dot{F}\{\bar{t}(\varepsilon), x[\bar{t}(\varepsilon), \varepsilon], u[\bar{t}(\varepsilon), \varepsilon]\}=0
$$

Equation (3) is the equation which defines the time $\bar{t}(\varepsilon)$ on each trajectory. Of course, this condition does not uniquely define $\bar{t}(\varepsilon)$ since it holds also at relative minima and other relative maxima of $F[x(t)]$. However, in the numerical implementation of the necessary conditions, this difficulty may be easily avoided by monitoring the value of $F[x(t)]$.

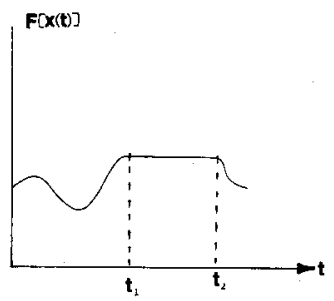

Fig. 2 Minimax with a nonunique maximum (or flat). 
With respect to the continuity assumption on the control vector we shall show that there exist two basic cases: 1 ) If either $\dot{F}$ does not depend explicitly upon $u$ or $\dot{F}$ contains $u$ explicitly with the maximum of $F$ occuring on a flat (i.e., Fig. 2), then the results may be applied without modification to problems in which the class of admissible controls is piecewise continuous. 2) If $\dot{F}$ contains $u$ explicitly and the maximum of $F$ is unique (i.e., Fig. 1), then the method (with a suitable modification) is applicable only to problems in which $u^{*}(t)$ is continuous in a neighborhood of $\bar{t}$. (The reason for this is that if $u(\bar{t})$ is undefined, then $\dot{F}$ need not be zero at $\bar{t}$. In any case, $\dot{F}\left(\bar{t}^{-}\right) \geqq 0$ and $\dot{F}\left(\bar{t}^{+}\right) \leqq 0$.) In the following sections the reasons for these continuity assumptions will become apparent.

Finally, in Refs. 1-4, problems of the following form are considered.

$$
\begin{array}{lc}
\text { Minimize: } & J=\max _{\substack{t \varepsilon\left[t_{0}, t\right] \\
\text { Subject to: }}} F[x(t)] \\
\text { constraints }
\end{array}
$$

Note that this class of problems does not include problems in which end-point and path functions are also desirable in the performance index. Since the physical problems which motivated this analysis must include such terms, the following problem will be considered in this paper.

Minimize:

Subject to:

$$
J=K F[x(\bar{t})]+\phi\left(t_{f}, x_{f}\right)+\int_{t_{0}}^{t} f_{L} L(t, x, u) d t
$$

$$
\begin{gathered}
x=f(t, x, u), \quad x\left(t_{0}\right)=x_{0} . \quad(n-\mathrm{vec}) \\
\pi\left(t_{f}, x_{f}\right)=0 \quad(\bar{p}-\mathrm{vec}) \\
u \varepsilon U \quad(u, m-\mathrm{vec})
\end{gathered}
$$

where $K$ is a specified nonnegative constant and $\bar{t}_{\varepsilon}\left(t_{0}, t_{f}\right)$ is the time (or first time) at which $F[x(t)]$ achieves its maximum value on $\left[t_{0}, t_{f}\right]$. Note that the classical Bolza problem is defined by $K=0$, and the pure minimax problem is defined when $K=1$, $\phi=0$, and $L=0$.

\section{Necessary Conditions for Minimax Problems with a Unique Maximum}

In this section we shall consider minimax problems which possess optimal solutions of the type shown in Fig. 1, i.e., the maximum of $F[x(t)]$ is attained at only one point on $\left[t_{0}, t_{f}\right]$, say $\bar{t} \varepsilon\left(t_{0}, t_{f}\right)$. To treat the problem within the framework of the classical calculus of variations, we must assume for now that $F$ does not depend upon $u$ explicitly, i.e.,

$$
d F / d t=F_{x}^{T}[x] f[t, x, u]=\dot{F}[t, x]
$$

Then, $\dot{F}[t, x(t)]$ is a continuous function since $x(t)$ is continuous and $\dot{F}[\bar{t}, x(\bar{t})]=0$ may be adjoined to the functional $J$ in Eq. (5) with a constant multiplier $\mu$. That is, the minimax problem is defined by Eqs. (5-8) and the intermediate point constraint

$$
\dot{F}[\bar{t}, x(\bar{t})]=0
$$

It is shown in Appendix $\mathbf{A}$ that the first-order necessary conditions for the minimax problem defined by Eqs. $(5-8,10)$ consist of the usual Euler-Lagrange and transversality conditions (at $t_{f}$ ) along with the following "jump conditions" at $\bar{t}$

$$
\begin{gathered}
\lambda\left(\bar{t}^{+}\right)=\lambda\left(\bar{t}^{-}\right)-\left(K F_{x}+\mu \dot{F}_{x}\right)_{\bar{t}} \\
H\left(\bar{t}^{+}\right)=H\left(\bar{t}^{-}\right)+\mu \dot{F}_{t}(\bar{t})
\end{gathered}
$$

As noted previously, these conditions are similar to Eqs. (3.13.4) and (3.13.5) of Ref. 7, which are true at the entry point of the bounded subarc (or touch point) in an SVIC problem.

Now consider the case when components of $u$ appear explicitly in $\dot{F}$. The main difficulty with this case is that control variables are not required to be continuous; at most, they are usually only required to be piecewise continuous. With regard to the first variation and the associated requirement that

$$
(d \dot{F} / d \varepsilon)\{\bar{t}(\varepsilon), x[\bar{t}(\varepsilon), \varepsilon], u[\bar{t}(\varepsilon), \varepsilon]\}=0
$$

the terms $\dot{F}_{t}, \dot{F}_{x}$, and $\dot{F}_{u}$ may not be defined at $\bar{t}$ if $u\left(\bar{t}^{-}\right) \neq u\left(\bar{t}^{+}\right)$, which is an admissible case if the class of controls is assumed to be the set of piecewise continuous functions on $\left[t_{0}, t_{f}\right]$. Thus, to treat this case, some of the control variables have to be assumed continuous in a neighborhood of $\bar{t}$, and this is equivalent to treating them as state variables with their derivatives as new control variables. Note that only the control variables which appear explicitly in Eq. (13) need to be treated in this manner, and these controls only need to be assumed continuous in a neighborhood of $\bar{t}$; that is, they may be piecewise continuous on other portions of the trajectory.

With respect to the analogy with SVIC problems, an SVIC $S(t, x) \geqq 0$ is said to be of order $p$ if $S^{(p)} \equiv d^{p} S / d t^{p}$ is the derivative of $S(t, x)$ which first contains the control explicitly in the case of a scalar control. Thus, an order can be associated with the function $F[x(t)]$ as follows

Definition: Let $u_{i}$ be a scalar control. The minimax function $F[x(t)]$ is said to be of order $p$ with respect to the control $u_{i}$ if $d^{p} F / d t^{p}$ is the derivative of $F$ which first contains $u_{i}$ explicitly.

With this definition it follows that a minimax function of order greater than one requires no modification of the control variable, and the analysis of appendix $A$ is applicable immediately. An interesting possibility is that a large class of minimax functions of odd order greater than one may possess only unique relative maxima (as opposed to flats) since a corresponding property is true for odd ordered SVIC problems. ${ }^{9}$

\section{Application to Re-Entry Heating Problems}

To fix the ideas of the previous section and Appendix A, the results will be interpreted in terms of a re-entry heating problem. Consider a re-entry problem in which three major effects are to be included in the performance index: a) high cross range, b) low insulation weight, which is a function of total heating (an integral effect); and c) low skin weight, which is a function of the maximum heating rate that occurs during entry (an intermediate point effect).

One way of treating this problem is to include the effects of items $a$ and $b$ in the performance index and impose an inequality constraint on heating rate. If it is desirable to avoid the inequality constraint, the problem could be treated in an approximate manner by adjoining

$$
P_{1} \int_{t_{0}}^{t} \ddot{q}(x, u)^{2} d t, \quad\left(P_{1}>0, \quad \text { penalty coefficient }\right)
$$

to the performance index (in addition to the cross-range and total heating terms). This would tend to "flatten out" $q$ and distribute the peak heating, while the total heating term would guard against too large of a trade of maximum heating rate for total heating.

An alternative to these methods is to attack the problem directly as a minimax problem as described in the previous section. Assuming that

$$
q=C_{q} \rho(r)^{1 / 2} V^{3}
$$

where $C_{q}$ is a constant heat rate coefficient, we wish to minimize the following performance index

$$
J=K C_{q} \rho(\bar{r})^{1 / 2} V(\bar{t})^{3}-r_{e} \tilde{\phi}_{f}+P \int_{t_{0}}^{t_{f}} C_{q} \rho(r)^{1 / 2} V^{3} d t
$$

where for this section the optimal solution is assumed to possess a unique maximum heating rate. The equations of motion are:

$$
\begin{gathered}
\dot{r}=V \sin \gamma ; \quad \dot{\theta}=V \cos \gamma \cos \psi / r \cos \tilde{\phi} \\
\dot{\tilde{\phi}}=V \cos \gamma \sin \psi / r ; \quad \dot{V}=-\left(k \sin \gamma / r^{2}\right)-D(h, V, \alpha) / m(17) \\
\dot{\gamma}=-k \cos \gamma / r^{2} V+V \cos \gamma / r+[\tilde{L}(h, V, \alpha) / m V] \cos u_{1} \\
\dot{\psi}=-V \cos \gamma \cos \tilde{\psi} \sin \tilde{\phi} / r \cos \phi-[\tilde{L}(h, V, \alpha) / m V] \sin u_{1} / \cos \gamma \\
\dot{\alpha}=u_{2}
\end{gathered}
$$

Here the angle of attack $\alpha$ is treated as a state variable or a constant; that is, if $\alpha$ is treated as a control, then the heating rate is a first-order minimax term with respect to $\alpha$ because $\dot{V}$ de- 
pends explicitly upon $\alpha$. The only requirement is that $\alpha$ be continuous in a neighborhood of $\bar{t}$; if cesired, the $\alpha$ equation could be dropped and $\alpha \equiv \mathrm{u}_{2}$, with the continuity restriction in the neighborhood of $\bar{t}$ only. (In this case, $d u_{2}(\bar{t})$ would be treated as an independent state variation in the development of the associated necessary conditions.)

With regard to the bank angle $u_{1}$ heating rate is a second-order minimax term since neither $\dot{V}$ nor $\dot{r}$ contain $u_{1}$ explicitly. Thus, with bank angle and angle-of-attack rate as the control variables, the analysis of the previous section is applicable. The variational Hamiltonian for the problem is

$$
H=P C_{q} \rho(r)^{1 / 2} V^{3}+\sum_{i=1}^{7} \lambda_{i} f_{i}(t, x, u)
$$

where $\dot{r} \equiv f_{1}, \ldots, \dot{\alpha} \equiv f_{7}$. The minimax function is

$$
F=C_{q} \rho(r)^{1 / 2} V^{3}
$$

and

$$
\begin{array}{r}
\dot{F}=\frac{1}{2} C_{q} \rho(r)^{-1 / 2}(\partial \rho / \partial r)(V \sin \gamma) V^{3}+3 C_{q} \rho(r)^{1 / 2} V^{2} \\
{\left[-k \sin \gamma / r^{2}-D(h, V, \alpha) / m\right]}
\end{array}
$$

As will be shown, this problem can be attacked with a gradienttype method without introducing any new variables (i.e., the multiplier $\mu$ ) if it is so desired.

Let $u^{(0)}(t)$ be an initial control estimate. Integrate the equations of motion forward to some cutoff condition and monitor the value of $\dot{q}\left(h^{(0)}, V^{(0)}\right)$. Logic can be developed to save three consecutive sate vectors, the second of which achieves the maximum sampled data value of $q$ on the $x^{(0)}$-trajectory. (This can be accomplished with storage reserved for four state vectors during the forward integration.) Denote these states by $x^{(0)}\left(t_{1}\right), x^{(0)}\left(t_{2}\right)$, and $x^{(0)}\left(t_{3}\right)$.

Assuming that the terminal conditions are adjoined to the performance index by penalty functions, the adjoint variables are defined at $t_{f}^{(0)}$ by

$$
\lambda^{(0)}\left(t_{f}\right)=\partial J /\left.\partial x_{f}\right|^{(0)}
$$

Integrate the adjoint equations backwards until $t=t_{3}$. Determine by any means the point $\bar{t}^{(0)} \varepsilon\left(t_{1}, t_{3}\right)$ at which the maximum $\dot{q}^{(0)}(t)$ occurs and integrate (or interpolate) to this point, which will be denoted as $\bar{t}^{+}$. We now wish to choose a $\lambda\left(\bar{t}^{-}\right)$which causes the $d x(\bar{t})$ and $d \bar{t}$ terms to vanish in the expression for $\delta J$. [Note that we have the values of $\lambda\left(\bar{t}^{+}\right)$(by integration), $u\left(\bar{t}^{-}\right)$, and $u\left(\bar{t}^{+}\right)$.] Since we will insure that $F\left(\bar{t}^{(J)}\right)=0$ on each iterate, this can be accomplished by satisfying Eqs. (A17) and (A18) of Appendix A. Since $\dot{F}$ does not depend upon $t$ explicitly, these equations become

$\left[K \dot{F}_{x}^{T}(\bar{t})-\lambda^{T}\left(\bar{t}^{-}\right)+\lambda^{T}\left(\bar{t}^{+}\right)\right] d x(\bar{t})+\left[H\left(\bar{t}^{-}\right)-H\left(\bar{t}^{+}\right)\right] d \bar{t}=0$

$$
\dot{F}_{x}^{T}(\bar{t}) d x(\bar{t})=0
$$

In terms of problem variables, Eq. (22) is the differential form which results by taking the differential of Eq. (20). The resultant expression is the following form

$$
B_{1} \cos \gamma d \gamma+B_{2} d r+B_{3} d V+B_{4} d \alpha=0
$$

Assuming $\gamma \neq \pm \pi / 2, d \gamma$ may be determined as a function of the remaining differentials, say,

$$
d \gamma=A_{1} d r+A_{2} d V+A_{3} d \alpha
$$

where the $A_{i}$ are well-defined functions of the state variables at $\bar{t}$. Upon substitution of Eq. (24) into Eq. (21), in terms of problem variables, the following differential form is obtained:

$$
\begin{aligned}
& \left\{\left[\frac{1}{2} K C_{q} \rho^{-1 / 2}(\partial \rho / \partial r) V^{3}\right]_{\bar{t}}+\Delta \lambda_{1}(\bar{t})\right\} d r+\Delta \lambda_{2}(\bar{t}) d \theta+\Delta \lambda_{3}(\bar{t}) \dot{d} \phi \\
& +\left[\left(3 K C_{q} \rho^{1 / 2} V^{2}\right)_{t}+\Delta \lambda_{4}(\bar{t})\right] d V+\Delta \lambda_{5}(\bar{t})\left(A_{1} d r+A_{2} d V+A_{3} d \alpha\right) \\
& \quad+\Delta \lambda_{6}(\bar{t}) d \psi+\Delta \lambda_{7}(\bar{t}) d \alpha+\left[H\left(\bar{t}^{-}\right)-H\left(\bar{t}^{+}\right)\right] d \bar{t}=0
\end{aligned}
$$

where

$$
\Delta \lambda_{i}(\bar{t}) \equiv \lambda_{i}\left(\bar{t}^{+}\right)-\lambda_{i}\left(\bar{t}^{-}\right) \quad(i=1, \ldots, 7)
$$

Since Eq. (24) has been employed to eliminate the dependence among the differentials at $\bar{t}$, Eq. (25) is a differential form in independent differentials and, thus, the coefficients must vanish. We obtain immediately that $\lambda_{2}, \lambda_{3}$, and $\lambda_{6}$ are continuous at $\bar{t}$; that is,

$$
\lambda_{i}\left(\bar{t}^{-}\right)=\lambda_{i}\left(\bar{t}^{+}\right) \quad(i=2,3,6)
$$

Upon solution of the remaining four equations defined by the vanishing coefficients, we obtain

$$
\begin{gathered}
\lambda_{5}\left(\bar{t}^{-}\right)=\left\{(V \sin \gamma)_{\bar{t}} Q_{1}+\dot{V}(\bar{t}) Q_{2}-\dot{\gamma}\left(\bar{t}^{+}\right) \lambda_{5}\left(\bar{t}^{+}\right)\right. \\
-\lambda_{6}\left(\bar{t}^{+}\right)\left[\dot{\psi}\left(\bar{t}^{+}\right)-\dot{\psi}\left(\bar{t}^{-}\right)\right] \\
\left.-\lambda_{7}\left(\bar{t}^{+}\right)\left[\dot{\alpha}\left(\bar{t}^{+}\right)-\dot{\alpha}\left(\bar{t}^{-}\right)\right]+Q_{3} \lambda_{5}\left(\bar{t}^{+}\right)\right\} /\left[Q_{3}-\dot{\gamma}\left(\bar{t}^{-}\right)\right] \\
\lambda_{1}(\bar{t})=\lambda_{1}\left(\bar{t}^{+}\right)+A_{1} \Delta \lambda_{5}(\bar{t})+Q_{1} \\
\lambda_{4}\left(\bar{t}^{-}\right)=\lambda_{4}\left(\bar{t}^{+}\right)+A_{2} \Delta \lambda_{5}(\bar{t})+Q_{2} \\
\lambda_{7}\left(\bar{t}^{-}\right)=\lambda_{7}\left(\bar{t}^{+}\right)+A_{3} \Delta \lambda_{5}(\bar{t})
\end{gathered}
$$

where

$$
\begin{gathered}
Q_{1} \equiv\left[\frac{1}{2} K C_{q} \rho^{-1 / 2}\left(\partial \rho^{\prime} \partial r\right) V^{3}\right]_{\bar{t}} \\
Q_{2} \equiv\left(3 K C_{q} \rho^{1 / 2} V^{2}\right)_{\bar{t}} \\
Q_{3} \equiv(V \sin \gamma)_{\bar{t}} A_{1}+\dot{V}(\bar{t}) A_{2}+\dot{\alpha}\left(\bar{t}^{-}\right) A_{3}
\end{gathered}
$$

The variables $\lambda_{i}\left(\dot{\bar{t}}^{-}\right)$are now well defined and the numerical integration may proceed to $t=t_{0}$. The gradient is then determined and a gradient step can be defined. The same procedure holds for the other iterates, and the scheme can be used with a conjugate gradient method as well as the classical gradient method. Also, again noting the analogy with SVIC problems, the computational method discussed in Ref. 10 also applies to this problem since both problems have basically the same type of necessary conditions. If a shooting method is desired, cases $1,2,3$, of Appendix A may be employed.

\section{Necessary Conditions for Minimax Problems with a Flat Maximum}

This section discusses the development of necessary conditions for the minimax problem defined by the performance index of Eq. (5) when the optimal trajectory possesses a "flat" with respect to the minimax function $F[x(t)]$ (see Fig. 2). Let $x^{*}(t)$, $t \varepsilon\left[t_{0}, t_{f}\right]$, be a trajectory which minimizes $J$ of Eq. (5), and suppose

$$
\max _{t \in\left[t_{0}, t_{f}\right]} F\left[x^{*}(t)\right]=F\left[x^{*}\left(t_{1}\right)\right]
$$

$F\left[x^{*}(t)\right]=F\left[x^{*}\left(t_{1}\right)\right], t \in\left[t_{1}, t_{2}\right], t_{2} \neq t_{1},\left[t_{1}, t_{2}\right] \subset\left[t_{0}, t_{f}\right]$

that is, the maximum value of $F$ occurs at every point on a nonzero interval.

Since $F\left[x^{*}(t)\right] \equiv F\left[x^{*}\left(t_{1}\right)\right]$ on $\left[t_{1}, t_{2}\right]$, all time derivatives of $F\left[x^{*}(t)\right]$ vanish on $\left(t_{1}, t_{2}\right)$. Again in analogy with the SVIC problem, suppose $u$ is a scalar control and let $F[x(t)]$ be a $p$ th order minimax function with respect to $u$. The vector control case will be discussed later. Then, since $x(t)$ is continuous at $t_{1}$, it follows that

$$
\dot{F}\left[x^{*}\left(t_{1}\right)\right]=\ldots=F^{(p-1)}\left[x^{*}\left(t_{1}\right)\right]=0
$$

and the control which keeps $F$ constant on $\left[t_{1}, t_{2}\right]$ may be determined from

$$
F^{(p)}[t, x, u]=0 \quad\left[\text { on }\left(t_{1}, t_{2}\right)\right]
$$

To determine the necessary conditions for this problem, first suppose $p>1$. Equation (37) represents intermediate point constraints, and Eq. (38) is used to determine $u(t, x)$ on $\left(t_{1}, t_{2}\right)$. Thus, the following augmented functional is defined below. Minimize:

$$
\begin{array}{r}
J=K F\left[x\left(t_{1}\right)\right]+v^{T} N\left[t_{1}, x\left(t_{1}\right)\right]+\phi\left(t_{f}, x_{f}\right) \\
+\int_{t_{0}}^{t_{1}^{-}}\left[H-\lambda^{T} \dot{x}\right] d t+\left.\int_{t_{1}^{+}}^{t_{\overline{2}}^{-}}\left[H-\lambda^{T} \dot{x}\right]\right|_{F^{(p)}=0} d t \\
+\int_{t_{2}^{+}}^{t f}\left[H-\lambda^{T} \dot{x}\right] d t
\end{array}
$$

Subject to:

$$
\begin{gathered}
H \equiv L(t, x, u)+\lambda^{T} f(t, x, u), \quad \dot{x}=f(t, x, u) \\
x\left(t_{0}\right)=x_{0}, \pi\left(t_{f}, x_{f}\right)=0
\end{gathered}
$$




$$
\begin{gathered}
N\left[t_{1}, x\left(t_{1}\right)\right] \equiv\left[\begin{array}{c}
\dot{F}\left[t_{1}, x\left(t_{1}\right)\right] \\
\vdots \\
F^{(p-1)}\left[t_{1}, x\left(t_{1}\right)\right]
\end{array}\right]=0 \\
F^{(p)}[t, x(t), u(t)]=0, \quad t \varepsilon\left(t_{1}, t_{2}\right) .
\end{gathered}
$$

where $v$ is a $p-1$ vector of constant Lagrange multipliers and $K$ is a specified positive constant. As shown in Appendix B, the following jump conditions at $t=t_{1}$ are necessary conditions for a minimum:

$$
\begin{gathered}
\lambda\left(t_{1}^{+}\right)=\lambda\left(t_{1}^{-}\right)-\left[K F_{x}+\left(v^{T} N\right)_{x}\right]_{t_{1}} \\
H\left(t_{1}^{+}\right)=H\left(t_{1}^{-}\right)+v^{T} N_{t_{1}}
\end{gathered}
$$

Also, for the case of a scalar control variable the Euler-Lagrange equations are

$$
\dot{\lambda}=\left\{\begin{array}{l}
-H_{x}, \quad t \varepsilon\left[t_{0}, t_{1}\right), \quad t \varepsilon\left(t_{2}, t_{f}\right] \\
-H_{x}-g_{x}, \quad t \varepsilon\left(t_{1}, t_{2}\right)
\end{array}\right.
$$

where $g_{x}$ is obtained from the following function

$$
F^{(p)}(t, x, u)=0 \Rightarrow u=g(t, x)
$$

For the problem of multiple control variables, the only change in the necessary conditions is in Eq. (46) and the equations for the controls on $\left(t_{1}, t_{2}\right)$. For example, suppose two control variables, $u_{1}$ and $u_{2}$, are present. Without loss of generality, $F^{(p)}$ will take one of the following forms

$$
F^{(p)}\left(t, x, u_{1}, u_{2}\right)
$$

or

$$
F^{(p)}\left(t, x, u_{1}\right) \quad\left[\text { or } F^{(p)}\left(t, x, u_{2}\right)\right]
$$

The only term which is affected by the additional control variable in the first variation [Eq. (B2)] is

$$
\left.\int_{t_{1}^{+}}^{t_{2}}\left[\left(H_{x}+\dot{\lambda}\right)^{T} \delta x+H_{u}^{T} \delta u\right]\right|_{F(p)=0} d t
$$

In the case of Eq. (48), one of the control variable variations may be determined as a function of variations in the state and the other control variable, for example,

$$
\delta u_{2} \equiv A \delta u_{1}+B^{T} \delta x
$$

which is then substituted into Eq. (50); that is,

$$
\int_{t_{1}^{+}}^{t_{2}^{-}}\left[\left(H_{x}+\dot{\lambda}+H_{u_{2}} B\right)^{T} \delta x+\left(H_{u_{1}}+H_{u_{2}} A\right) \delta u_{1}\right] d t
$$

Now, $\delta \mathrm{x}$ and $\delta \mathrm{u}_{1}$ may be treated as independent variations, and on $\left(t_{1}, t_{2}\right)$

$$
\dot{\lambda}=-\mathrm{H}_{x}-\mathrm{H}_{u_{2}} \mathrm{~B}
$$

In the case of interior controls on the subarc $\left(t_{1}, t_{2}\right)$, the following equations are used to solve for $u_{1}, u_{2}$ :

$$
\left.\begin{array}{l}
H_{u_{1}}+A H_{u_{2}}=0 \\
F^{(q)}\left[t, x, u_{1}, u_{2}\right]=0
\end{array}\right\} \quad \Rightarrow u_{1}, u_{2}\left[\text { on }\left(t_{1}, t_{2}\right)\right]
$$

In the case of Eq. (49), $F^{(p)}$ depends explicitly upon only one of the two controls, say $u_{1}$. Then, $\delta u_{1}$ is a function of $\delta x$ whereas $\delta u_{2}$ is "free" (subject to normality considerations). Thus,

$$
\delta u_{1}=C^{T} \delta x
$$

and the equation corresponding to Eq. (52) above is

$$
\int_{t_{1}^{+}}^{t_{2}^{-}}\left[\left(H_{x}+\dot{\lambda}+H_{u_{1}} C\right)^{T} \delta x+H_{u_{2}} \delta u_{2}\right] d t
$$

which can be used to form equations analogous to Eqs. (53-55). Similar results may be developed for higher dimensional control vectors.

Finally, consider the $p=1$ case and assume $u$ is a scalar. The generalization to the vector control case is similar to the extension given above for the $p>1$ case. With a flat, nonunique maximum instead of a unique maximum, no problem is encountered with the $p=1$ case; that is, one need not require continuity of some or all control variables at $t_{1}$, because $\dot{F}$ is not required to be continuous at $t_{1}$ for the determination of the first variation.
Thus, the only difference between the $p=1$ and $p>1$ cases for a flat nonunique maximum is that the $p=1$ case does not have any intermediate point constraints at $t_{1}$ (and thus no Lagrange multipliers, $v_{i}$ ).

\section{A Simple Example}

The necessary conditions of the previous section will be applied to a simple example first discussed by Barry. ${ }^{4}$

$$
\begin{array}{cc}
\text { Minimize: } & J=\max _{t e[0,5]} x_{2}(t) \\
\text { Subject to }: \quad \dot{x}_{1}=x_{2}, \quad x_{1}(0)=-4, \quad x_{2}(0)=0 \\
\dot{x}_{2}=u, \quad x_{1}(5)=0, \quad x_{2}(5)=0 ; \quad|u| \leqq 1
\end{array}
$$

Because of the tight boundary condition constraints, one can determine the solution by inspection. The optimal control is

$$
u^{*}(t)=\left\{\begin{array}{rr}
+1 & t \varepsilon[0,1) \\
0 & t \varepsilon(1,4) \\
-1 & t \varepsilon(4,5]
\end{array}\right.
$$

and the minimax value of $x_{2}(t)$ is achieved at each $t \varepsilon[1,4]$ with $x_{2}(t)=1, t \varepsilon[1,4]$, i.e., the maximum is a flat maximum. Thus, the necessary conditions of the previous section are applicable.

In analogy with the SVIC problem, ${ }^{7}$ consider the three regions: Region $\mathrm{I}=\left\{t \varepsilon\left[0, t_{1}\right)\right\}$, Region II $=\left\{t \varepsilon\left(t_{1}, t_{2}\right)\right\}$, Region III $=$ $\left\{t \varepsilon\left(t_{2}, 5\right]\right\}$. For this problem

$$
\begin{gathered}
H=\lambda_{1} x_{2}+\lambda_{2} u \\
\dot{F}=x_{2}=u \Rightarrow u=0 \quad \text { (on flat) }
\end{gathered}
$$

The Euler-Lagrange equations are the same in each region because $u=0$ in Region II (in general, the multiplier-equations may be different in a region where $F^{(p)} \equiv 0$ )

$$
\dot{\lambda}_{1}=0, \quad \dot{\lambda}_{2}=-x_{2}
$$

The multipliers and Hamiltonian in the neighborhood of $t_{1}$ are defined by Eqs. (44-45) (where $N$ is a zero-dimension vector for this problem since $F[x]=x_{2}$ is a first-order $(p=1)$ minimax function)

$$
\begin{gathered}
\lambda_{1}\left(t_{1}^{+}\right)=\lambda_{1}\left(t_{1}^{-}\right), \quad \lambda_{2}\left(t_{1}^{+}\right)=\lambda_{2}\left(t_{1}^{-}\right)-1 \\
H\left(t_{1}^{+}\right)=H\left(t_{1}^{-}\right)
\end{gathered}
$$

The multipliers and Hamiltonian are continuous elsewhere by the Weierstrass-Erdmann corner conditions.

Equations (59) and (63) may be integrated easily in each of the regions, and after applying Eqs. (64), a system of four equations $\left[x_{1}(5)=0, x_{2}(5)=0, H\left(t_{1}^{+}\right)=H\left(t_{1}^{-}\right), H\left(t_{2}^{+}\right)=H\left(t_{2}^{-}\right)\right]$is used to solve for the four unknowns $\left.\left[\lambda_{1}(0), \lambda_{2}(0), t_{1}, t_{2}\right)\right]$. The multiplier solutions are

$$
\begin{aligned}
& \lambda_{1}(t) \equiv \lambda_{10}=-1 / 3 \\
& \lambda_{2}(t)= \begin{cases}-(1-t) / 3 & t \varepsilon[0,1) \\
-(1-t) / 3-1 & t \varepsilon(1,5]\end{cases}
\end{aligned}
$$

The method gives the correct solution, and the multiplier vector is the same as Barry's, which is obtained as the limit of a sequence of approximate problems.

\section{Conclusions}

A method for attacking directly minimax problems which arise in trajectory optimization has been developed. Problems of interest which can be treated by this technique are, among others, a) determination of the minimum peak heating rate in re-entry, b) determination of the minimum peak deceleration in re-entry, and c) determination of the minimum peak bending moment or dynamic pressure in ascent. The method is developed in such a way that other quantities can be included in the performance index (e.g., total heating and cross range in items a and b, and payload weight in item c). The analysis indicates a close relationship between minimax problems and problems with state variable inequality constraints, and this fact is used to define 
numerous numerical algorithms for the iterative solution of minimax problems.

The method is applicable to 1) all problems in which the first time derivative of the minimax function does not contain control variables explicitly, and 2) all problems with a "flat" maximum (including problems in which the first time derivative of the minimax function contains control variables). It should be noted that a numerically oriented formal procedure is used to derive the necessary conditions, and in this procedure the form of the solution must be assumed beforehand (e.g., peak or flat maximum). If the true optimal is not of the form assumed, then, in analogy with the results obtained in Ref. 9 (Sec. 8) for SVIC problems, the multipliers employed in the augmented functionals (A3) and/or (B1) are not guaranteed by a multiplier rule and false minima may result. However, since there are only two possibilities for the extremal in the minimax problem, this fact should not cause undue anxiety.

\section{Appendix A: Necessary Conditions for a Unique Maximum}

Consider the case when $\mathrm{F}$ does not contain $u$ explicitly; that is,

$$
(d / d t) F[x \bar{t})]=\dot{F}[\bar{t}, x(\bar{t})]
$$

At $\bar{t}(\varepsilon)$ it is necessary that

$$
F[\bar{t}(\varepsilon), x(\bar{t}(\varepsilon), \varepsilon)]=0
$$

and this condition can be considered as an intermediate boundary condition. Thus, we consider the following problem.

Minimize:

$$
\begin{aligned}
& J=K F[x(\bar{t})]+\mu \dot{F}[\bar{t}, x(\bar{t})]+\phi\left(t_{f}, x_{f}\right) \\
& +\int_{t_{0}}^{\bar{t}^{-}}\left[H-\lambda^{T} \dot{x}\right] d t+\int_{\bar{t}^{+}}^{t_{f}}\left[H-\lambda^{T} \dot{x}\right] d t
\end{aligned}
$$

Subject to :

$$
\begin{gathered}
H \equiv L(t, x, u)+\lambda^{T} f(t, x, u), \quad \dot{x}=f(t, x, u) \\
x\left(t_{0}\right)=x_{0}, \quad \pi\left(t_{f}, x_{f}\right)=0 \\
\dot{F}[\bar{t}, x(\bar{t})]=0
\end{gathered}
$$

Let $x^{*}(t)$ be a relative minimum for $J$ and suppose there do not exist any corners on the subarcs $\left(t_{0}, \bar{t}\right)$ and $\left(\bar{t}, t_{f}\right)$. The first variation of $J$ is then

$$
\begin{aligned}
\delta J= & K F_{x}^{T}(\bar{t}) d x(\bar{t})+\mu \dot{F}_{\bar{t}}(\bar{t}) d \bar{t}+\mu \dot{F}_{x}^{T}(\bar{t}) d x(\bar{t}) \\
& +d \phi-\left[\lambda^{T} \delta x\right]_{\bar{t}^{-}}+\left[\lambda^{T} \delta x\right]_{\bar{t}^{+}}+\left[H-\lambda^{T} x\right]_{\bar{t}^{-}} d \bar{t} \\
- & {\left[H-\lambda^{T} \dot{x}\right]_{\bar{t}^{+}} d \bar{t}+\int_{t_{0}}^{\bar{t}^{-}}\left[\left(H_{x}+\dot{\lambda}\right)^{T} \delta x+H_{u}^{T} \delta u\right] d t } \\
& +\int_{\bar{t}^{+}}^{t_{j}}\left[\left(H_{x}+\dot{\lambda}\right)^{T} \delta x+H_{u}^{T} \delta u\right] d t
\end{aligned}
$$

On each subarc the usual Euler-Lagrange equations hold, and the only new terms result from the variations at $\bar{t}$. Noting that from Ref. 7

$$
d x(\bar{t})=\delta x\left(\bar{t}^{-}\right)+\dot{x}\left(\bar{t}^{-}\right) d \bar{t}=\delta x\left(\bar{t}^{+}\right)+\dot{x}\left(\bar{t}^{+}\right) d \bar{t}
$$

the terms outside of the integral in Eq. (A7) must vanish, and thus, $K F_{x}^{T}(\bar{t}) d x(\bar{t})+\mu \dot{F}_{t}(\bar{t}) d \bar{t}+\mu \dot{F}_{x}^{T}(\bar{t}) d x(\bar{t})$

$$
-\lambda^{T}\left(\bar{t}^{-}\right)\left[d x(\bar{t})-\dot{x}\left(\bar{t}^{-}\right) d \bar{t}\right]+\lambda^{T}\left(\bar{t}^{+}\right)\left[d x(\bar{t})-\dot{x}\left(\bar{t}^{+}\right) d \bar{t}\right]
$$

$+\left[H\left(\bar{t}^{-}\right)-\lambda^{T}\left(\bar{t}^{-}\right) \dot{x}\left(\bar{t}^{-}\right)\right] d \bar{t}-\left[H\left(\bar{t}^{+}\right)-\lambda^{T}\left(\bar{t}^{+}\right) \dot{x}\left(\bar{t}^{+}\right)\right]=0 \quad$ (A9) Collecting coefficients of the independent differentials $d x(\bar{t})$ and $d \bar{t}$

$$
\begin{aligned}
{\left[\left(K F_{x}^{T}+\right.\right.} & \left.\left.\mu \dot{F}_{x}^{T}\right)_{\bar{t}}-\lambda^{T}\left(\bar{t}^{-}\right)+\lambda^{T}\left(\bar{t}^{+}\right)\right] d x(\bar{t}) \\
& +\left[\mu \dot{F}_{t}(\bar{t})+\lambda^{T}\left(\bar{t}^{-}\right) \dot{x}\left(\bar{t}^{-}\right)-\lambda^{T}\left(\bar{t}^{+}\right) \dot{x}\left(\bar{t}^{+}\right)+H\left(\bar{t}^{-}\right)\right. \\
& \left.-\lambda^{T}\left(\bar{t}^{-}\right) \dot{x}\left(\bar{t}^{-}\right)-H\left(\bar{t}^{+}\right)+\lambda^{T}\left(\bar{t}^{+}\right) \dot{x}\left(\bar{t}^{+}\right)\right] d \bar{t}=0
\end{aligned}
$$

Then, the jump conditions at $\bar{t}$ are

$$
\begin{gathered}
\lambda\left(\bar{t}^{+}\right)=\lambda\left(\bar{t}^{-}\right)-\left(K F_{x}+\mu \dot{F}_{x}\right)_{\bar{t}} \\
H\left(\bar{t}^{+}\right)=H\left(\bar{t}^{-}\right)+\mu \dot{F}_{t}(\bar{t})
\end{gathered}
$$

Let us now consider how one might use these equations in a shooting method to demonstrate that we have the proper number of equations for the unknows. We shall consider three possible implementations of a shooting method.

\section{Case 1}

Guess $\lambda_{0}^{(0)}, \bar{t}^{(0)}, \mu^{(0)}, t_{f}^{(0)}\left(n+3\right.$ unknowns). Integrate to $\bar{t}^{(0)}$ and evaluate $\dot{F}\left(\bar{t}^{(0)}\right)$. Jump the multipliers as defined in Eq. (A11). Determine $u\left(\bar{t}^{+}\right)$from $H_{u}\left[\bar{t}, x(\bar{t}), \lambda\left(\bar{t}^{+}\right), u\left(\bar{t}^{+}\right)\right]=0$ and then determine $\dot{x}\left(\bar{t}^{+}\right)=f\left[\bar{t}, x(\bar{t}), u\left(\bar{t}^{+}\right)\right]$. Evaluate the terms in Eq. (A12) and record the residual. Integrate to $t_{f}$ from $\bar{t}^{+}$with $\lambda\left(\bar{t}^{+}\right)$ and evaluate $M\left[t_{f}, x_{f}, \lambda_{f}\right]$ (the terminal and transversality conditions). The new values of $\lambda_{0}, \bar{t}, \mu$ are determined by the residuals in

$$
\begin{gathered}
(1) \quad \dot{F}\left[\bar{t}^{(0)}, x^{(0)}\left(\bar{t}^{(0)}\right)\right] \\
\text { (1) } \quad H\left(\bar{t}^{(0)+}\right)-H\left(\bar{t}^{(0)-}\right)-\mu^{(0)} \dot{F}_{t}\left(\bar{t}^{(0)}\right) \\
(n+1) \quad M\left[t_{f}^{(0)}, x_{f}^{(0)}, \lambda_{f}^{(0)}\right]
\end{gathered}
$$

Thus, we have $n+3$ equations to determine the $n+3$ unknowns.

\section{Case 2}

Guess $\lambda_{0}^{(0)}, \mu^{(0)}, t_{f}^{(0)}\left(n+2\right.$ unknowns). Integrate until $\dot{F}\left[x^{(0)}(t)\right]$ $=0$ and designate this point as $\bar{t}^{(0)}$. Thus, on each iterate the constraint $\dot{F}[x(\bar{t})]=0$ will be satisfied. Performing the same evaluations as in case 1 , the $n+2$ equations for the $n+2$ unknowns are:

$$
\text { (1) } \begin{gathered}
H\left(\bar{t}^{+}\right)-H\left(\bar{t}^{-}\right)-\mu \dot{F}_{t}(\bar{t})=0 \\
(n+1) \quad M\left(t_{f}, \lambda_{f}, x_{f}\right)=0
\end{gathered}
$$

\section{Case 3}

Since $\dot{F}[x(\bar{t})]=0$ is satisfied on each trajectory, one can eliminate the multiplier $\mu$ if it is so desired. In this case, only $\lambda_{0}$ and $t_{f}$ would be involved in the iteration scheme. The necessary conditions for this case result by considering the following problem. Minimize:

$$
J=K F[x(\bar{t})]+\phi\left(t_{f}, x_{f}\right)+\int_{t_{0}}^{t_{f}}\left[H-\lambda^{T} \dot{x}\right] d t
$$

Subject to:

$$
\begin{gathered}
d \pi\left(t_{f}, x_{f}\right)=0 \\
d \dot{F}[\bar{t}, x(\bar{t})] \equiv \dot{F}_{t} d \bar{t}+\dot{F}_{x}^{T} d x(\bar{t})=0
\end{gathered}
$$

After forming the first variation and eliminating all terms except those at $\bar{t}$, we have:

$$
\begin{gathered}
{\left[K F_{x}^{T}(\bar{t})-\lambda^{T}\left(\bar{t}^{-}\right)+\lambda^{T}\left(\bar{t}^{+}\right)\right] d x(\bar{t})+\left[H\left(\bar{t}^{-}\right)-H\left(\bar{t}^{+}\right)\right] d \bar{t}=0} \\
\dot{F}_{t}(\bar{t}) d t+\dot{F}_{x}^{T}(\bar{t}) d x(\bar{t})=0
\end{gathered}
$$

Suppose that $t$ appears explicitly in $\dot{F}$ and that $\dot{F}_{t} \neq 0$. (If this is not the case, one could make a similar assumption about one of the $x_{i}^{\prime}$ 's; see the re-entry heating section). Then,

$$
d \bar{t}=-\left(\dot{F}_{t}^{-1} \dot{F}_{x}^{T}\right)_{\bar{t}} d x(\bar{t})
$$

Upon substitution into Eq. (A17)

$$
\begin{gathered}
\left\{K F_{x}^{T}(\bar{t})-\lambda^{T}\left(\bar{t}^{-}\right)+\lambda^{T}\left(\bar{t}^{+}\right)+\left(\dot{F}_{t}^{-1} F^{F}\right)_{t}\left[H\left(\bar{t}^{+}\right)-H\left(\bar{t}^{-}\right)\right]\right\} \\
d x(\bar{t})=0
\end{gathered}
$$

The coefficients of $d x(\bar{t})$ in Eq. (A20) represent $n$ equations in the $n$ unknowns $\lambda\left(\bar{t}^{-}\right)$if one is integrating backward or $\lambda\left(t^{+}\right)$if one is integrating forward. Note that Eq. (A20) may not be linear in the unknown $\lambda\left(\bar{t}^{+}\right)$since $u$ (which occurs in $H$ ) may be a nonlinear function of $\lambda$. Thus the utility of case 3 in a shooting technique is problem dependent.

\section{Appendix B: Necessary Conditions for a Nonunique Maximum}

Consider a $p>1$ minimax problem with a nonunique maximum. The only difference between the development of the necessary 
conditions for this class of minimax problems and SVIC problems is that $K F\left[x\left(t_{1}\right)\right]$ appears in the minimax problem (with $K$ specified and $F\left[x\left(t_{1}\right)\right]$ in general nonzero) while a product of an unknown constant multiplier, say $v_{1}$, and a quantity which is zero on the boundary, say $S(t, x)$, that is $v_{1} S\left[t_{1}, x\left(t_{1}\right)\right]$, appears in the SVIC problem. Thus, we should expect the necessary conditions to be quite similar to the SVIC necessary conditions.

The functional to be minimized here is

$$
\begin{aligned}
& J=K F\left[x\left(t_{1}\right)\right]+v^{T} N\left[t_{1}, x\left(t_{1}\right)\right]+\phi\left(t_{f}, x_{f}\right)+\int_{t_{0}}^{t_{1}^{-}}\left[H-\lambda^{T} \dot{x}\right] d t \\
& +\quad \int_{t_{1}^{+}}^{t_{2}^{-}}\left[H-\lambda^{T} \dot{x}\right] d t+\int_{t_{2}^{+}}^{t_{f}}\left[H-\lambda^{T} \dot{x}\right] d t
\end{aligned}
$$

Let $x^{*}(t)$ be a relative minimum for $J$ and suppose there do not exist any corners on the subarcs $\left(t_{0}, t_{1}\right),\left(t_{1}, t_{2}\right),\left(t_{2}, t_{f}\right)$. Assume that $\mathrm{u}$ is a scalar. The first variation of $J$ is

$$
\begin{aligned}
\delta J= & K F_{x}\left(t_{1}\right) d x\left(t_{1}\right)+v^{T} N_{t_{1}} d t_{1}+\left(v^{T} N\right)_{x_{1}}^{T} d x\left(t_{1}\right) \\
& +d \phi-\left[\lambda^{T} \delta x\right]_{t_{1}}+\left[\lambda^{T} \delta x\right]_{t_{1}^{+}}-\left[\lambda^{T} \delta x\right]_{t_{2}}+\left[\lambda^{T} \delta x\right]_{t_{2}^{+}} \\
+ & {\left[H-\lambda^{T} \dot{x}\right]_{t_{1}} d t_{1}-\left[H-\lambda^{T} \dot{x}\right]_{t_{1}^{+}} d t_{1}+\left[H-\lambda^{T} \dot{x}\right]_{t_{2}} d t_{2} } \\
& -\left[H-\lambda^{T} \dot{x}\right]_{t_{2}^{+}} d t_{2}+\int_{t_{0}}^{t_{1}^{-}}\left[\left(H_{x}+\dot{\lambda}\right)^{T} \delta x+H_{u} \delta u\right] d t \\
+ & \int_{t_{1}^{+}}^{t^{2}}\left[\left(H_{x}+\dot{\lambda}+g_{x}\right)^{T} \delta x\right] d t+\int_{t_{2}^{+}}^{t_{f}}\left[\left(H_{x}+\dot{\lambda}\right)^{T} \delta x+H_{u} \delta u\right] d t
\end{aligned}
$$

where $g_{x}$ is defined by

$$
\begin{gathered}
F^{(p)}[t, x, u]=0 \Rightarrow u=g(t, x) \\
\delta u=g_{x}^{T} \delta x
\end{gathered}
$$

The usual Euler-Lagrange equations hold on the subarcs $\left[t_{0}, t_{1}\right)$ and $\left(t_{2}, t_{f}\right]$, while

$$
\dot{\lambda}=-H_{x}-g_{x}\left[\text { on }\left(t_{1}, t_{2}\right) \text { only }\right]
$$

on the subarc on which $F[x(t)]=$ const. The other equations of interest result from the variations at $t_{1}$ and $t_{2}$, and noting that $d x\left(t_{i}\right)=\delta x\left(t_{i}^{-}\right)+\dot{x}\left(t_{i}^{-}\right) d t_{i}=\delta x\left(t_{i}^{+}\right)+\dot{x}\left(t_{i}^{+}\right) d t_{i}, \quad(i=1,2)$

the following differential form must vanish:

$$
\begin{gathered}
K F_{x}\left(t_{1}\right) d x\left(t_{1}\right)+v^{T} N_{t_{1}} d t_{1}+\left(v^{T} N\right)_{x}^{T} d x\left(t_{1}\right) \\
-\lambda^{T}\left(t_{1}^{-}\right) d x\left(t_{1}\right)+\lambda^{T}\left(t_{1}^{+}\right) d x\left(t_{1}\right)-\lambda^{T}\left(t_{2}^{-}\right) d x\left(t_{2}\right) \\
+\lambda^{T}\left(t_{2}^{+}\right) d x\left(t_{2}\right)+H\left(t_{1}^{-}\right) d t_{1}-H\left(t_{1}^{+}\right) d t_{1} \\
+H\left(t_{2}^{-}\right) d t_{2}-H\left(t_{2}^{+}\right) d t_{2}=0
\end{gathered}
$$

After collecting like differentials, the coefficients must vanish and the following necessary conditions result:

$$
\lambda\left(t_{1}^{+}\right)=\lambda\left(t_{1}^{-}\right)-\left[K F_{x}+\left(v^{T} N\right)_{x}\right]_{t_{1}}
$$

$$
H\left(t_{1}^{+}\right)=H\left(t_{1}^{-}\right)+v^{T} N_{t_{1}}
$$

The Lagrange multipliers and Hamiltonian are continuous everywhere else on the interval $\left[t_{0}, t_{f}\right]$.

Again, to demonstrate that we have the proper number of equations for the unknowns, let us consider a possible implementation or a shooting method for the $p>1$ case.

Guess:

$$
\lambda_{0}^{(0)}, t_{1}^{(0)}, t_{2}^{(0)}, t_{f}^{(0)}, v^{(0)}[n+3+(p-1) \text { unknowns }]
$$

Integrate forward to $t_{1}^{(0)}$, jump the multipliers at $t_{1}$, as defined by Eq. (B8), and record the residuals of Eq. (B9) and Eq. (42). Integrate from $t_{1}^{(0)}$ to $t_{2}^{(0)}$ using the control which satiesfies Eq. (B3) and the multiplier equation (B5). At $t_{2}^{(0)}$ record the residuel in

$$
H\left(t_{2}^{+}\right)-H\left(t_{2}^{-}\right)
$$

which should be continuous at $t_{2}$. Proceed from $t_{2}^{(0)}$ to $t_{f}^{(0)}$ and record the residuals in the $n+1$ terminal conditions. The $n+3+(p-1)$ residuals may then be used to update the initial parameter estimates.

\section{References}

${ }^{1}$ Johnson, C. D., "Optimal Control with Chebyshev Minimax Performance Index, ASME Transactions on Basic Engineering, Ser. D, Vol. 89, No. 2, June 1967, pp. 251-262.

${ }^{2}$ Shilov, A. A. and Zhelnin, Y. N., "The Minimum of the Maximum Deceleration in Spacecraft Reentry," Cosmic Research, Vol. 4, No. 4, July-Aug. 1966 , pp. 552-557.

${ }^{3}$ Michael, G. J., "Computation of Chebycheff Optimal Control," AIAA Journal, Vol. 9, No. 5, May 1971, pp. 973-975.

${ }^{4}$ Barry, P. E., "Optimal Control with Minimax Cost," IEEE Transactions on Automatic Control, Vol. AC-16, Aug. 1971, pp. 354357.

${ }^{5}$ Warga, J., "Minimizing Variational Curves Restricted to a Preassigned Set," Transactions of American Mathematical Society, Vol. 112, 1964, pp. 432-455.

${ }^{6}$ Warga, J., "Minimax Problems and Unilateral Curves in the Calculus of Variations," Journal of SIAM on Control, Ser. A, Vol. 3 No. 1, 1965, pp. 91-105.

${ }^{7}$ Bryson, A. E. and Ho., Y. C., Applied Optimal Control, Blaisdell, Waitham, Mass., 1969, Chaps. 3 and 9.

${ }^{8}$ Denham, W. F., "Steepest Ascent Solution of Optimal Programming Problems," Ph. D. thesis, 1963, Harvard Univ., Cambridge, Mass.

${ }^{9}$ Jacobson, D. H., Lele, M. M., and Speyer, J. L., "New Necessary Conditions of Optimality for Control Problems with State-Variable Inequality Constraints," Journal of Mathematical Analysis and Applications, Vol. 35, No. 2, Aug. 1971, pp. 255-284.

${ }^{10}$ Denham, W. F. and Bryson, A. E., "Optimal Programming Problems with Inequality Constraints II: Solution by SteepestAscent," AIAA Journal, Vol. 2, No. 1, Jan. 1964, pp. 25-34. 